PROCEEDINGS OF THE

AMERICAN MATHEMATICAL SOCIETY

Volume 130, Number 8, Pages 2313-2318

S 0002-9939(02)06352-9

Article electronically published on January 17, 2002

\title{
ROOTS OF COMPLEX POLYNOMIALS AND WEYL-HEISENBERG FRAME SETS
}

\author{
PETER G. CASAZZA AND NIGEL J. KALTON
}

(Communicated by David R. Larson)

\begin{abstract}
A Weyl-Heisenberg frame for $L^{2}(\mathbb{R})$ is a frame consisting of modulates $E_{m b} g(t)=e^{2 \pi i m b t} g(t)$ and translates $T_{n a} g(t)=g(t-n a), m, n \in \mathbb{Z}$, of a fixed function $g \in L^{2}(\mathbb{R})$, for $a, b \in \mathbb{R}$. A fundamental question is to explicitly represent the families $(g, a, b)$ so that $\left(E_{m b} T_{n a} g\right)_{m, n \in \mathbb{Z}}$ is a frame for $L^{2}(\mathbb{R})$. We will show an interesting connection between this question and a classical problem of Littlewood in complex function theory. In particular, we show that classifying the characteristic functions $\chi_{E}$ for which $\left(E_{m} T_{n} \chi_{E}\right)_{m, n \in \mathbb{Z}}$ is a frame for $L^{2}(\mathbb{R})$ is equivalent to classifying the integer sets $\left\{n_{1}<n_{2}<\cdots<n_{k}\right\}$ so that $f(z)=\sum_{j=1}^{k} z^{n_{i}}$ does not have any zeroes on the unit circle in the plane.
\end{abstract}

\section{INTRODUCTION}

A family of vectors $\left(f_{i}\right)$ in a Hilbert space $H$ is called a frame for $H$ if there are constants $A, B>0$ so that

$$
A\|f\|^{2} \leq \sum_{i}\left|\left\langle f, f_{i}\right\rangle\right|^{2} \leq B\|f\|^{2} \text {, for all } f \in H .
$$

We call $A$ (resp. $B$ ) a lower (resp. upper) frame bound of the frame. The largest $A$ and the smallest $B$ which work in (1.1) above are called the optimal frame bounds. If $P$ is an orthogonal projection on $H$, and $\left(f_{i}\right)$ is a frame for $H$ with frame bounds $A, B$, then for all $f \in P H$ we have

$$
A\|f\|^{2} \leq \sum_{i}\left|\left\langle f, f_{i}\right\rangle\right|^{2}=\left.\sum_{i}\left\langle f, P f_{i}\right\rangle\right|^{2} \leq B\|f\|^{2} .
$$

It follows that $\left(P f_{i}\right)$ is a frame for $P H$ with the same frame bounds $A, B$.

An important class of frames used in signal/image processing, data compression etc. are the Weyl-Heisenberg frames. For $f \in L^{2}(\mathbb{R})$ and $a, b$ real numbers we define translation by a (resp. modulation by b) by $T_{a} f(t)=f(t-a)$ (resp. $\left.E_{b} f(t)=e^{2 \pi i b t} f(t)\right)$. For a fixed $g \in L^{2}(\mathbb{R})$, we say that $(g, a, b)$ generates a WeylHeisenberg frame (WH-frame for short) if $\left(E_{m b} T_{n a} g\right)_{m, n \in \mathbb{Z}}$ forms a frame for $L^{2}(\mathbb{R})$. A fundamental question in this area is to explicitly represent those families $(g, a, b)$ which generate Weyl-Heisenberg frames for $L^{2}(\mathbb{R})$. Much work has been done on this question by Ron and Shen [13, 14], Janssen [10], Casazza, Christensen

Received by the editors February 28, 2000 and, in revised form, February 16, 2001.

1991 Mathematics Subject Classification. Primary 30C15, 11C08, 42C15, $46 \mathrm{C} 05$.

The first author was supported by NSF DMS 9706108 and the second author by NSF DMS 9870027. 
and Janssen [3], Casazza and Lammers 44, and a host of other authors. The papers [4, 10] concentrate on the question of finding the characteristic functions $\chi_{E}$ so that $\left(\chi_{E}, a, b\right)$ generates a Weyl-Heisenberg frame. Janssen [10] has made quite a detailed study of this question, and one consequence of his work is that even this very special case of the general problem is quite a deep question. It is known 7] that $(g, 1,1)$ generates a WH-frame if and only if $\left(E_{m} T_{n} g\right)$ is a Riesz basis for $L^{2}(\mathbb{R})$. In this paper we will show that the question of classifying the characteristic functions $\chi_{E}$ so that $\left(\chi_{E}, 1,1\right)$ generates a Weyl-Heisenberg frame is equivalent to a classical problem of Littlewood in complex function theory. This shows that this question in WH-frame theory is even more difficult than previously thought, as well as giving important connections between frame theory and complex function theory.

Now we will pass to Littlewood's problem. In 1968 Littlewood 11 studied the class of functions $\mathcal{A}_{n}$ which consists of polynomials of the form $\sum_{i=1}^{n} a_{i} z^{i}$, with $a_{i} \in\{0,1\}$. On page 25 of [11] he writes: "These raise some fascinating questions." One of the main questions raised by Littlewood is:

Problem 1.1 (Littlewood). Classify the integer sets $\left\{n_{1}<n_{2}<\cdots<n_{k}\right\}$ so that $f(z)=\sum_{j=1}^{k} z^{n_{i}}$ does not have any zeroes on the unit circle in the plane.

There is a huge literature on the zeroes of polynomials in $\mathcal{A}_{n}$ as well as various other related classes (e.g. where the coefficients come from the set $\{-1,0,1\}$ ). For an up to date view of this subject, we refer the reader to [1, 2, 12] and their references.

\section{MAin RESUlts}

We need the Zak transform (called the kq-representation by Zak and also called the Weil-Brezin map in the literature) but brought to the level of an "art form" by Janssen (see [5, 8, 9]). We define the Zak transform to be the unitary mapping $Z(\cdot)$ from $L^{2}(\mathbb{R})$ onto $L^{2}\left([0,1]^{2}\right)$ that takes the orthonormal basis $\left\{E_{m} T_{n} \chi_{[0,1]}\right)_{m, n \in \mathbb{Z}}$

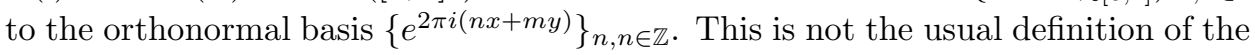
Zak transform, but is an equivalent formulation [7]. It is known (see [7, 8, 9]) that

$$
Z g(x, y)=\sum_{n \in \mathbb{Z}} g(x+n) e^{2 \pi i n y}, \text { for all } x, y \in[0,1],
$$

and

$$
Z\left(E_{m} T_{n} g\right)(x, y)=e^{2 \pi i(n x+m y)} Z g(x, y) .
$$

Now we have the following (somewhat well-known) result.

Proposition 2.1. Let $E$ be a measurable subset of $[0,1], F=\bigcup_{n \in \mathbb{Z}}(E+n)$ and $g \in L^{2}(F)$. The following are equivalent:

(1) $\left(E_{m} T_{n} g\right)_{m, n \in \mathbb{Z}}$ is a frame for $L^{2}(F)$ with optimal frame bounds $A, B$.

(2) We have

$$
\begin{aligned}
0<A & =\underset{(x, y) \in E \times[0,1]}{\operatorname{essinf}}|Z g(x, y)|^{2} \\
& \leq \operatorname{essiup}_{(x, y) \in E \times[0,1]}|Z g(x, y)|^{2}=B<\infty
\end{aligned}
$$


Proof. Since $Z(\cdot)$ is a unitary operator, the frame bounds for $\left(E_{m} T_{n} g\right)$ are the same as the frame bounds for $\left(e^{2 \pi i(n x+m y)} Z g(x, y)\right)$. Also, by equation (2.1) and the definition of $E, F$, we have

$$
Z g(x, y)=0, \text { for all } x \notin E .
$$

Since $\left(e^{2 \pi i(n x+m y)}\right)_{m, n \in \mathbb{Z}}$ is an orthonormal basis for $L^{2}\left([0,1]^{2}\right)$, we have for any $F(x, y) \in L^{2}\left([0,1]^{2}\right)$,

$$
\begin{gathered}
\sum_{m, n \in \mathbb{Z}}\left|\left\langle F(x, y), e^{2 \pi i(n x+m y)} Z g(x, y)\right\rangle\right|^{2} \\
=\|F(x, y) \overline{Z g(x, y)}\|_{L^{2}(E \times[0,1])}^{2} .
\end{gathered}
$$

It follows that the upper (resp. lower) frame bound for $\left(e^{2 \pi i(n x+m y)} Z g(x, y)\right)$ is the square of the norm of the multiplication operator (resp. the inverse of the multiplication operator) $F \rightarrow F \cdot Z g$ on $L^{2}(E \times[0,1])$. A direct calculation shows that these norms are precisely the bounds given in (2) of the proposition.

We now have

Proposition 2.2. Let $E_{i} \subset[0,1], F_{i}=\bigcup_{n \in \mathbb{Z}}\left(E_{i}+n\right), F=\bigcup_{i} F_{i}$, and assume $F_{i} \cap F_{j}=\emptyset$, for all $i \neq j$. Let $g_{i}$ be a function supported on $F_{i}$ and assume that $g=\sum_{i} g_{i} \in L^{2}(\mathbb{R})$. The following are equivalent:

(1) $\left(E_{m} T_{n} g\right)_{m, n \in \mathbb{Z}}$ is a frame for $L^{2}(F)$ with optimal frame bounds $A, B$.

(2) For each $i,\left(E_{m} T_{n} g_{i}\right)_{m, n \in \mathbb{Z}}$ is a frame for $L^{2}\left(F_{i}\right)$ with optimal frame bounds $A_{i}, B_{i}$, and $0<A=\inf A_{i} \leq \sup B_{i}=B<\infty$.

Proof. (1) $\Rightarrow(2)$ : Let $P_{i}$ be the orthogonal projection of $L^{2}(F)$ onto $L^{2}\left(F_{i}\right)$ given by $P_{i} f=\left.f\right|_{F_{i}}$. Now, $P_{i} g=g_{i}$, and, as we observed in the introduction, $\left(g_{i}, 1,1\right)$ generates a WH-frame for $L^{2}\left(F_{i}\right)$ with frame bounds $A, B$. Hence, $A \leq A_{i} \leq B_{i} \leq$ $B$.

$(2) \Rightarrow(1)$ : By Proposition 2.1 and our assumptions in (2), for each $i$ we have

$$
\begin{aligned}
A & \leq A_{i} \leq \underset{(x, y) \in F_{i} \times[0,1]}{\operatorname{essinf}}\left|Z g_{i}(x, y)\right|^{2} \\
& \leq \underset{(x, y) \in F_{i} \times[0,1]}{\operatorname{ess} \operatorname{up}_{i}}\left|Z g_{i}(x, y)\right|^{2} \leq B_{i} \leq B .
\end{aligned}
$$

Now, $Z g=\sum_{i} Z g_{i}$ and by our assumption that $F_{i} \cap F_{j}=\emptyset$, for all $i \neq j$, we have

$$
\text { support } Z g_{i} \cap \text { support } Z g_{j}=\emptyset \text {, for all } i \neq j \text {. }
$$

It follows that

$$
\underset{(x, y) \in F \times[0,1]}{\operatorname{ess} \sup }|Z g(x, y)|^{2}=\sup _{i} \operatorname{esssup}_{(x, y) \in F_{i} \times[0,1]}\left|Z g_{i}(x, y)\right|^{2}=\sup _{i} B_{i}=B .
$$

Similarly,

$$
A=\inf _{i} A_{i}=\operatorname{essinf}_{(x, y) \in F \times[0,1]}|Z g(x, y)|^{2} .
$$

It follows that $(g, 1,1)$ generates a WH-frame for $L^{2}(F)$ with frame bounds $A, B$ by Proposition 2.1

Now we have a constructive characterization of certain Weyl-Heisenberg frame sets for $a=b=1$. To simplify the notation, we call a measurable subset $F \subset \mathbb{R}$ a Weyl-Heisenberg frame set for $(a, b)$ if $\left(\chi_{F}, a, b\right)$ generates a Weyl-Heisenberg frame for $L^{2}(\mathbb{R})$. 
Theorem 2.3. Fix integers $n_{1}<n_{2}<\cdots<n_{k}$. The following are equivalent:

(1) The set $F=\bigcup_{j=1}^{k}\left([0,1)+n_{j}\right)$ is a Weyl-Heisenberg frame set for $(1,1)$ with frame bounds $A, B$.

(2) We have $A \leq\left|\sum_{j=1}^{k} z^{n_{j}}\right|^{2} \leq B$, for all $|z|=1$.

(3) For every measurable set $E \subset[0,1]$ of positive measure, and for the set $F_{0}=\bigcup_{j=1}^{k}\left(E+n_{j}\right),\left(E_{m} T_{n} \chi_{F_{0}}\right)_{m, n \in \mathbb{Z}}$ is a frame for $L^{2}\left(F_{0}\right)$ with frame bounds $A, B$.

Proof. In Proposition 2.1, if $g=\chi_{F}$, then

$$
Z g(x, y)=\chi_{[0,1]}(x) \sum_{j=1}^{k} e^{2 \pi i n_{j} y}=\sum_{j=1}^{k} e^{2 \pi i n_{j} y}, \text { for all } x, y \in[0,1] \text {. }
$$

Hence,

$$
A \leq \operatorname{essinf}_{(x, y) \in[0,1]^{2}}|Z g(x, y)|^{2} \leq \operatorname{ess~sup}_{(x, y) \in[0,1]^{2}}|Z g(x, y)|^{2} \leq B
$$

if and only if for all $|z|=1$,

$$
A \leq\left|\sum_{j=1}^{k} z^{n_{j}}\right|^{2} \leq B
$$

This proves the equivalence of (1) and (2), which is clearly implied by (3).

We now show that $(1)$ implies $(3)$. By $(1),\left(\chi_{F}, 1,1\right)$ generates a WH-frame for $L^{2}(F)$ with frame bounds $A, B$. Let $P$ be the orthogonal projection of $L^{2}(F)$ onto $L^{2}\left(F_{0}\right)$ given by $P f=\left.f\right|_{F_{0}}$. Then $P \chi_{F}=\chi_{F_{0}}$, and, as we have observed in the introduction, it follows that $\left(\chi_{F_{0}}, 1,1\right)$ generates a WH-frame for $L^{2}\left(F_{0}\right)$ with frame bounds $A, B$.

We call a measurable set $F \subset \mathbb{R}$ an elementary A-Weyl-Heisenberg frame set of length $\mathbf{k}$ if $F=\bigcup_{j=1}^{k}\left(E+n_{j}\right)$ for some $\left(n_{j}\right)$ and some measurable subset $E$ in $[0,1)$ of positive measure and we have

$$
A \leq \inf _{|z|=1}\left|\sum_{j=1}^{k} z^{n_{j}}\right|^{2}
$$

We end with our classification of all WH-frame sets for $a=b=1$. We first note that for $F \subset \mathbb{R}$, functions in the span of $\left(E_{m} T_{n} g\right)_{m, n \in \mathbb{Z}}$ have their support in $\bigcup_{n \in \mathbb{Z}}(F+n)$. Hence, a necessary condition for $F$ to be a WH-frame set for $(1,1)$ is that $\left|\mathbb{R}-\bigcup_{n}(F+n)\right|=0$.

Theorem 2.4. Let $F$ be a subset of $\mathbb{R}$ for which $\left|\mathbb{R}-\bigcup_{n \in \mathbb{Z}}(F+n)\right|=0$ a.e. The following are equivalent:

(1) The set $F$ is a Weyl-Heisenberg frame set for $(1,1)$.

(2) There are constants $k, A>0$ so that $F=\bigcup_{i \in I} F_{i}$ ( $I$ is finite or infinite), where each $F_{i}$ is an elementary $A$-Weyl-Heisenberg frame set of length $\leq k$ and $\left(F_{i}+n\right) \cap\left(F_{j}+m\right)=\emptyset$ for all $i \neq j$ and all $m, n \in \mathbb{Z}$.

Proof. $(1) \Rightarrow(2)$ : Let $F$ be a WH-frame set. For each $j \in \mathbb{N}$ let $F_{j}=\{x \in[0,1)$ : $\#(x+\mathbb{N}) \cap F \#=j\}$, where \# denotes cardinality. A consequence of the WH-frame identity (see [7], Theorem 4.1.2, p. 648) is that $\sum_{n}\left|\chi_{F}(x+n)\right|^{2} \leq B$ a.e. Hence, 
there is a $k \in \mathbb{N}$ so that $\left|F_{j}\right|=0$, for all $j>k$. Now for $j \leq k$ and any finite set of integers $\zeta=\left\{n_{1}<n_{2}<\cdots<n_{j}\right\}$ let $F_{j, \zeta}=\left\{x \in F_{j}: x+n_{\ell} \in F, 1 \leq \ell \leq j\right\}$. The distinct non-empty $\left(F_{j, \zeta}\right)$ form a countable family of sets which are pairwise disjoint under translation by $n \in \mathbb{Z}$ and which by Proposition 2.2 satisfies (2).

$(2) \Rightarrow(1)$ : Fix $i$ and consider $\left(\chi_{F_{i}}, 1,1\right)$. Since $F_{i}$ is an elementary A-WH-frame set of length $k$, there are a $m \leq k$ and a set $E_{i} \subset[0,1]$ with $F_{i}=\bigcup_{j=1}^{m}\left(E_{i}+n_{j}\right)$ for some $n_{1}<n_{2}<\cdots<n_{m}$ so that for all $|z|=1$ we have

$$
A \leq\left|\bigcup_{j=1}^{m} z^{n_{j}}\right|^{2} \leq m \leq k .
$$

By Theorem $2.3\left(\chi_{F_{i}}, 1,1\right)$ generates a WH-frame with frame bounds $A, k$. Since the $\left(F_{i}\right)$ are disjoint, we have $\chi_{F}=\sum_{i} \chi_{F_{i}}$. Also, $\left(F_{i}+n\right) \cap\left(F_{j}+m\right)=\emptyset$, for all $i \neq j, \quad m, n \in \mathbb{Z}$, implies that $E_{i} \cap E_{j}=\emptyset$ for all $i \neq j$. Hence,

$$
|F|=\sum_{i}\left|F_{i}\right| \leq k \sum_{i}\left|E_{i}\right| \leq k
$$

and it follows that $\chi_{F} \in L^{2}(\mathbb{R})$. Now, by Proposition 2.2. $\left(\chi_{F}, 1,1\right)$ is a frame for $L^{2}(F)$ with frame bounds $A, k$.

\section{ACKNOWLEDGEMENT}

The authors would like to thank Tamas Erdélyi for some useful comments concerning the problems posed by Littlewood, and the referee for significant improvements to the clarity of the presentation in this paper.

\section{REFERENCES}

[1] P. Borwein and T. Erdélyi, On the zeroes of polynomials with restricted coefficients, Illinois Jour. Math. 41 (1997) 667-675. MR 98g:30008

[2] P. Borwein, T. Erdélyi, and G. Kós, Littlewood-type problems on [0,1], Proc. London Math. Soc. 379 (1999) 22-46. MR 2000c:11111]

[3] P.G. Casazza, O. Christensen and A.J.E.M. Janssen, Classifying tight Weyl-Heisenberg frames, The Functional and Harmonic Analysis of Wavelets and Frames, Baggett and Larson Edts, Contemp. Math 247, AMS, Providence, R.I. (1999) 131-148. MR 2001e:42036

[4] P.G. Casazza and M. Lammers, Classifying characteristic functions giving WeylHeisenberg frames, Proceedings SPIE, San Diego (2000).

[5] H.G. Feichtinger and T. Strohmer (eds.), Gabor Analysis and Algorithms: Theory and Applications, Birkhäuser, Boston (1998). MR 98h:42001

[6] D. Han and D. Larson, Frames, Bases and Group Representations, Memoirs AMS, Vol. 147, No. 697, Providence, RI, (2000). MR 2001a:47013

[7] C. Heil and D. Walnut, Continuous and discrete wavelet transforms, SIAM Review, 31 (4) (1989) 628-666. MR 91c:42032

[8] A.J.E.M. Janssen, Bergman transform, Zak transform, and coherent states, J. Math. Phys., 23 (5) (1982) 730-731. MR 84h:81041

[9] A.J.E.M. Janssen, The Zak transform: A signal transform for sampled time-continuous signals, Philips J. Res., 43 (1) (1988) 23-69. MR 89g:94005

[10] A.J.E.M. Janssen, Zak transforms with few zeros and the tie, preprint.

[11] J.E. Littlewood, Some Problems in Real and Complex Analysis, Heath Mathematical Monographs, Lexington, Massachusetts, 1968. MR 39:5777

[12] A. Odlyzko and B. Poonen, Zeroes of polynomials with 0,1 ceofficients, Ens. Math. Vol. 39 (1993), 317-348. MR 95b:11026 
[13] A. Ron and Z. Shen, Frames and stable bases for shift-invariant subspaces of $L_{2}\left(\mathbb{R}^{d}\right)$, Canadian Jour. of Math. 47 (5) (1995) 1051-1094. MR 96k:42049

[14] A. Ron and Z. Shen, Weyl-Heisenberg frames and Riesz bases in $L_{2}\left(\mathbb{R}^{d}\right)$, Duke Math. J. 89 (1997) 237-282. MR 98i:42013

Department of Mathematics, University of Missouri-Columbia, Columbia, Missouri 65211

E-mail address: pete@math.missouri.edu

Department of Mathematics, University of Missouri-Columbia, Columbia, Missouri 65211

E-mail address: nigel@math.missouri.edu 